\title{
La eficacia de las acciones de la Unión Africana para combatir el terrorismo en África. Los casos de Somalia y Nigeria
}

\section{Efficacy of African Union's actions to fight terrorism in Africa. The cases of Somalia and Nigeria}

\author{
Johanna Barrantes Sánchez. \\ Estudiante de Gestión de la Cooperación Internacional \\ Universidad Nacional, Costa Rica \\ johanna.barrantes.sanchez@est.una.ac.cr \\ Keisell Córdoba Bazán \\ Estudiante de Relaciones Internacionales, \\ Universidad Nacional, Costa Rica \\ keysell.cordoba.bazan@est.una.ac.cr \\ Karla Rodríguez Villafuerte \\ Estudiante de Gestión de la Cooperación Internacional \\ Universidad Nacional, Costa Rica \\ karla.rodriguez.villafuerte@est.una.ac.cr
}

\begin{abstract}
Resumen
En el presente artículo se aborda la problemática del terrorismo en África. Se parte de los antecedentes de este fenómeno en el continente para luego presentar dos casos de estudio, el grupo terrorista Al-Shabaab, localizado en Somalia, y el grupo BokoHaram en Nigeria. El objetivo del artículo es analizar la eficacia de la Unión Africana en la lucha contra el terrorismo a partir de su accionar en los dos casos de estudio, así como se busca evidenciar la alta incidencia de actores extrarregionales en el continente.
\end{abstract}

Palabras claves: Unión Africana, lucha contra el terrorismo, Somalia, Nigeria, Al-Shabaab, BokoHaram. 


\begin{abstract}
This article deals with the problem of terrorism in Africa. It parts from the background of this phenomenon in the continent, and then it presents two case studies, the terrorist group Al-Shabaab in Somalia and the group Boko Haram in Nigeria. The aim of this article is to analyze the efficacy of the African Union in the fight against terrorism, starting from the its actions in the two case studies, and it seeks to demonstrate the high incidence of extraregional actors in the continent.
\end{abstract}

Keywords: African Union, fight against terrorism, Somalia, Nigeria, AlShabaab, Boko Haram.

\section{Introducción}

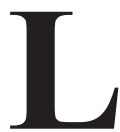

a creación de la Unión Africana (UA) en 2002 como sucesora de la Organización para la Unión Africana (OUA) ha llegado a tratar de mesurar las problemáticas que se han dado en torno a la seguridad y la estabilidad política en los países del continente, ya que la falta de incidencia que tuvo la OUA provocó que se reconfiguraran las prioridades, con el fin de establecer el desarrollo social y económico de África.

No obstante, para comenzar a pensar en una estabilidad económica o social se debe combatir uno de los principales detonantes de la falta de integración en la región, el terrorismo. Este ha sido provocado por diferencias étnicas y religiosas que llevan siglos en disputa, lo que ha causado un efecto en otros ámbitos de desarrollo del continente, ya que el carácter económico, político y social está vinculados para lograr una integración efectiva.

Por esta razón la UA tiene el mandato, como organismo supranacional, de facilitar el trabajo en conjunto entre los Estados miembros en la lucha contra el terrorismo. El presente artículo busca analizar la actuación de la Unión Africana con respecto al terrorismo, con el fin de determinar si existe incidencia de este organismo relativamente nuevo o si existe mayor influencia de otros actores.

Con el fin de una mejor comprensión, el artículo se divide en secciones. En la primera sección, se detalla información general de la Unión Africana, así como los factores que propiciaron la creación de esta, para conocer el contexto del organismo y comprender su accionar.

En la segunda sección, se destaca la experiencia africana con respecto al terrorismo, las causas de su intensificación en diferentes lugares de la región, con la finalidad de exponer la situación actual del continente, ya que el terrorismo no es una novedad sino un fenómeno que ha escalado a través del tiempo.

La tercera sección contiene información del funcionamiento de la Unión Africana 
y sus instituciones en materia de seguridad, a su vez, se menciona el papel de otros actores de carácter internacional que acompañan a la UA en su lucha contra el terrorismo y la inseguridad de los países africanos. De la misma forma, se hace mención a la fuente de financiamiento del organismo, que tiene un complicado panorama de fondos por parte de sus Estados miembros, ya que los problemas de pobreza de los países africanos son altos e incluso los países con mayor capacidad económica no se desligan de la desigualdad social y la economía poco sólida.

Con el objetivo de enfocar el accionar de la UA en el continente, se analizan, en la cuarta sección, dos casos de países que actualmente sufren con la presencia de grandes grupos terroristas, Al-Shabaab en Somalia y BokoHaram en Nigeria; estos afectan la región tanto a nivel interno como externo, debido a que han extendido su presencia a países fronterizos e inclusive actúan en conjunto con otras entidades terroristas como Al-Shabaab con el grupo Al Qaeda.

\section{Problemática}

El desequilibrio de poder entre el Estado y grupos insurgentes, que ocurre en África debido al creciente número de ataques terroristas ha puesto a las élites gobernantes en el continente a repensar las estrategias que se vienen tomando en la lucha contra el terrorismo. Sin embargo, se debe comprender que este fenómeno es antiguo y ha sido producto de una serie de coyunturas propias de África.

De la Unión Africana, al ser el organismo más cercano y mejor capacitado dentro del continente, se espera que mediante la cooperación de la totalidad de sus miembros, tome medidas conjuntas para atacar al terrorismo. No obstante, como ya se mencionó, recientemente se han dado muchos ataques terroristas, sin que la Unión Africana pueda responder a la amenaza de estos grupos. Lo que cuestiona el rol que ejerce el organismo en la eliminación de los ataques en contra de la seguridad civil.

Parte del reto que tiene África, ligado a lo que ya se mencionó, se relaciona con el combate a las organizaciones que se encuentran bien establecidas en cada uno de los países, para lo cual se necesita de un aparato de seguridad estatal fuerte que sea capaz de proteger a la población civil sin dejar de enfrentar el terrorismo doméstico para que no se vuelva regional. Este fenómeno no conoce fronteras, por tanto, la participación internacional debe ir en la línea de convertir los debilitados aparatos estatales africanos en gobiernos competentes y con las herramientas tanto técnicas, legales, como militares para erradicar el fenómeno terrorista.

\section{Antecedentes del terrorismo en África}

La preocupación de occidente por los grupos terroristas es notoria en fechas recientes, debido al aumento de los ataques terroristas en países europeos; sin embargo, existe un factor que debe considerarse al analizar este tipo de incidentes: los grupos terroristas como por ejemplo Al Qaida, los Talibanes y recientemente BokoHaram tienen bastantes años de haberse asentado en distintos países. Su organización logística, financiera y su presencia internacional es un proceso de larga data del siglo 
pasado. De hecho, si se observa la historia africana cercana, se notará cómo los primeros ataques de estos grupos, en un principio células terroristas, fueron hechos dentro de los límites de los países en los que estos grupos terroristas iniciaron (Pérez, 2004). Por tanto, en el presente apartado se pretende analizar la longevidad del fenómeno terrorista, la irrupción del terrorismo a occidente a inicios del presente siglo, algunos de los grupos terroristas más peligrosos y casos de países que más han tenido que luchar desde décadas pasadas contra la proliferación de estos.

Un primer punto por aclarar es que no todos los grupos terroristas siguen una misma línea de pensamiento o creencia; igualmente, en una misma región pueden existir varios grupos ideológicos utilizando el terrorismo para dominar sobre una mayoría. Por lo que hace muy difícil encasillarlos en una única corriente religiosa, incluso lo que pretenden lograr con el uso de métodos terroristas es distinto de un grupo a otro, por ejemplo, en el norte de África se encuentra muy diseminado el terrorismo islámico con ideología yihadista-salafista (Echeverría, s. f., p. 253). Asimismo, debe entenderse la existencia de lo que parece ser un primer esbozo de grupo terrorista del que degenerarían en el futuro muchas otras ramificaciones de grupos terroristas, conocida como los Hermanos Musulmanes, nacido en 1928. Posteriormente "extendería su influencia hacia el Este, hacia Oriente Próximo y Medio, dando lugar, entre otros productos, al embrión de lo que luego sería el Movimiento de Resistencia Islámico Palestino más conocido por su acrónimo de Hamás" (Echeverría, s. f., p. 254).
Es importante determinar, de igual forma, el periodo de inicio de los primeros ataques terroristas en África. En el caso del terrorismo islamista se daban golpes por los "Hermanos Musulmanes egipcios contra las autoridades del país, producidos ya incluso en los años cincuenta del pasado siglo, hasta los primeros atentados contra intereses turísticos en Túnez, a mediados de los años ochenta" (Echeverría, s. f., p. 252). También en la década de los sesenta en Nigeria se empezaron a dar genocidios entre comunidades vecinas, no obstante, existe el problema que todos los hechos anteriores en su momento no fueron analizados como ataques terroristas propiamente dichos, ya que se les veía como una "conflictividad endémica africana alimentada por factores como el mal trazado de fronteras, la interrelación obligada de etnias, clanes y grupos religiosos que no vivían tradicionalmente en armonía, la explotación de recursos, el mal reparto del poder" (Echeverría, s. f., p. 252). En las décadas subsecuentes, los ataques terroristas también se continuaron concentrando en el mismo continente africano y en lo que respecta al grupo terrorista de $\mathrm{Al}$ Qaida, en 1990 contaba ya con campos de entrenamiento en Sudán, utilizados además por otros grupos acólitos acogidos a su amplio paraguas financiero, entre ellos, al-Jihad, al-Gamma y al-Islamiya. Las primeras fatwas emitidas por al-Qaeda datan de 1992, en las que condena la presencia de tropas norteamericanas en Somalia. En 1993, al-Qaeda se responsabilizó de la muerte de 18 soldados estadounidenses en ese mismo país. En 1998 una nueva fat$w a$, procedente de ese grupo, estableció para todo musulmán el deber de matar "ciudadanos norteamericanos"; una fatwa 
considerada como la justificación religiosa que amparó la comisión de los atentados contra las embajadas de EEUU en Kenia y Tanzania (Pérez, 2004).

A partir del inicio del siglo XX, la concentración de ataques terroristas pasa de la zona africana a occidente, y es cuando se empieza a tomar seriamente la amenaza que representa, para la estabilidad del status quo internacional, el temor constante de un ataque terrorista. Este cambio es explicado, primeramente, porque la disputa en la región africana era para dominar sobre las dimensiones del contiene solamente, en otras palabras, los grupos terroristas de mayor permanencia tenían una "prioridad combatiente dirigida más contra otros musulmanes, a los que se considera desviados, heréticos o apóstatas, que contra Occidente" (Echeverría, s. f., p. 254). Es posible comprender esto, ya que se originó en su momento un conflicto entre un nacionalismo africano tendiente a rechazar las ideas provenientes del exterior. Esta corriente tiene sus raíces en un hombre llamado "SayyedQutb, ahorcado a fines de los años sesenta, es considerado como el primer teorizador moderno de la obligación de sublevarse contra los líderes musulmanes considerados «responsables» de propagar entre la comunidad musulmana o Umma los valores occidentales" (Echeverría, s. f., p. 254).

Todo lo anterior explica la evolución que se ha dado en cuanto a quién o quiénes los grupos terroristas, en diferentes épocas, señalan como sus enemigos $\mathrm{y}$, por tanto, contra los que deben arremeter; occidente, con su intervencionismo, también vino a romper la concepción de señalar al enemigo como el "infiel religioso", ahora se enfrentan a un enemigo distinto (occidente), que no puede luchar con sus mismos métodos porque tiene que cumplir con ciertas normas del derecho internacional.

Además, para el desarrollo del terrorismo, en aspectos como crecimiento en su alcance, letalidad en sus ataques y complejidad en sus redes, fue necesario que se destinaran grandes cantidades de dinero a la causa terrorista. Esto se logró con el involucramiento del crimen organizado, con los ingresos de las actividades de los grupos criminales dentro del entramado terrorista, por ejemplo, en "el caso de Al Qaeda en el Magreb Islámico es uno de los ejemplos existentes de sinergia entre ambos fenómenos. Las actividades criminales logran la financiación precisa para organizar acciones terroristas" (IEEE y IMDEP, 2013, p. 24). África, por sus condiciones geográficas y la debilidad de sus Estados (IEEE y IMDEP, 2013, p. 21), se convirtió en el terreno perfecto para el tráfico de drogas, personas, armas. Tomando el caso específico del tráfico de drogas se puede decir:

El tráfico de drogas es un medio habitual para la financiación de grupos y acciones terroristas. Parte de las actividades ilícitas desarrolladas en África Subsahariana y en el Sahel se traducen en fondos disponibles para Al Qaeda en el Magreb Islámico, al servicio de su presunta intención de convertirse en un actor de la yihad global. (IEEE y IMDEP, 2013, p. 21)

Por consiguiente, se dio una alianza entre los grupos terroristas y criminales que les ha permitido a los primeros diversificar sus 
fuentes de financiamiento e invirtir más, por tanto, en la innovación en sus ataques y en la tecnología (IEEE y IMDEP, 2013, p. 40), de tal manera que han logrado infiltrarse en muchos lugares de Occidente que se consideraban exentos del problema terrorista. En otras palabras, la adhesión de fondos criminales a la actividad terrorista, a pesar de que gran cantidad de miembros de grupos terroristas discuten sobre la moralidad de este dinero (IEEE y IMDEP, 2013), sin duda fue un factor decisivo que ha llevado al fenómeno terrorista de la región africana a sociedades de occidente, como se mencionó con anterioridad.

A pesar de todo lo anterior, la llegada del terrorismo africano a occidente no ha significado que haya mermado la cantidad de ataques terroristas en África; por el contrario, continúa estable el número de ataques $\mathrm{y}$, en algunos casos, puede que haya crecido. En la construcción de la amenaza del terrorismo han existido países que cuentan con un largo pasado en la lucha contra este, naciones que desde el siglo pasado vienen empleando medidas (políticas públicas y alianzas regionales e internacionales) para aplacar la difusión de la fiebre del terrorismo. En esta línea se van a presentar algunos casos en los que se podrá visualizar la evolución del terrorismo yihadista- salafista en estos países y la relativa calma con la que cuentan hoy en día, gracias a la persecución y procesamiento de los grupos terroristas.

Un primer caso es Libia, que durante los años 90, en el régimen de Muammar elGafafi sufría fuertes agresiones por los grupos terroristas, resulta contradictorio cómo el mismo dictador que apoyaba otros grupos terroristas en Europa intentaba eliminar la insurgencia en su país (Echeverría, s. f.). Por esto, en el periodo de 1995 y 1996 "el régimen del coronel Gadafi se vio obligado a llevar adelante una fuerte campaña antiterrorista en la zona oriental del país, con su epicentro en el triángulo formado por las ciudades de Benghazi, Tobruk y Darnah" (Echeverría, s. f., p. 259). Fruto de esto, "entre los años 1996 y 1997 pueden contabilizarse diversas operaciones contra los terroristas y a principios del año 1998 éstos se hacían visibles en los alrededores de Benghazi produciéndose varios enfrentamientos con las Fuerzas de Seguridad" (Echeverría, s. f., p. 259). Uno de los principales grupos que combatía contra el gobierno en Libia desde la década de los 80 era el Grupo Islámico Combatiente Libio (GICL) y continuó en operación hasta casi la primera década del presente siglo.

El embrión de ese GICL, que en el otoño de 2007 prestaba su fidelización a Al Qaeda, se manifestaba a lo largo de los años noventa a través de atentados contra policías y militares en la zona oriental del país, de orografía difícil y que fue el santuario de estos terroristas, pero también aquí la contundencia de las autoridades llevó a eclipsar el activismo de estos grupos y parte de sus dirigentes y cuadros empezaron pronto a ser más conocidos por su activismo fuera, en lugares lejanos como Afganistán, Pakistán o Irak, que dentro de las fronteras de su país. (Echeverría, s. f., p. 260).

Túnez es otro caso en el que el terrorismo fue un problema constante desde la década de los 80 hasta inicios del presente siglo. El fenómeno terrorista inicia por el 
descontento de los sectores más conservadores de la población por las políticas aplicadas por el líder de la revolución, en cuanto a la legislación a favor de los derechos de la mujer y la apertura del país hacia occidente (Echeverría, s. f.).

El grupo terrorista más representativo de la región, "el Movimiento de Tendencia Islámica (MTI) era a fines de los años ochenta un actor relevante si bien nunca llegó a ser legalizado ni con tal nombre ni con el posterior del HarkatEnnahdha" (Echeverría, s. f., p. 261). Dicho grupo intentó en 1990 ser declarado partido político, pero su solicitud fue rechazada por su involucramiento en el ataque contra la Agrupación Constitucional Democrática y pasa a ser perseguido, por lo que comienza con ataques terroristas en contra del sector turístico de Túnez.

Sus actividades terroristas continúan durante toda la década de los 90 y ya para el inicio del 2000, "Túnez fue uno de los escenarios más importantes del emergente terrorismo suicida que con la firma de Al Qaeda sucedió ya sin tregua a los macroatentados del 11-S en Estados Unidos" (Echeverría, s. f., p. 262). El régimen de Habib Burguiba luchó contra el grupo terrorista de MTI y otras células terroristas, lo que resultó en la desarticulación de muchos grupos terroristas, hasta conseguir que, alrededor del 2008:

la extremada vigilancia de las autoridades tunecinas dejan poco espacio para que los yihadistas-salafistas puedan actuar en el país, y ello explica tanto aquí como para el caso libio que los terroristas de dicha nacionalidad actúen con frecuencia más en el extranjero, es decir en la diáspora, que en su propio país. (Echeverría, s. f., p. 264)

Un último caso a exponer es Argelia, por su experiencia de más de dos décadas en cuanto a la lucha contra el terrorismo, que en la época de la disputa por la independencia tuvo un alza en los atentados. Se puede ubicar que, "a mediados de los años ochenta podemos situar ya la experiencia terrorista-guerrillera de los islamistas radicales comandados por Mustafá Bouyali bajo las siglas del Movimiento Islámico Armado (MIA), que realizaron ataques contra representantes del Estado" (Echeverría, s. f., p. 266).

Sin embargo, en esta coyuntura en específico se da una identificación del enemigo particular, ya que "la animadversión inicial de los islamistas radicales en Argelia se dirigía contra el socialismo y el comunismo y no contra Francia y Occidente: la aprobación de la Constitución de 1976 provocó ya los primeros conatos de contestación islamista" (Echeverría, s. f., p. 266). Durante todos los años de los 90 y 2000 se ha venido gestando una guerra del terrorismo dentro de Argelia, tanto así que ha habido una explosión de los atentados terroristas a partir del 2006, con especial énfasis en el 2008, uno de los años más sangrientos, y debe tomarse en cuenta que para el año 2007 se contabilizaron 500 muertes provocadas por ataques terroristas (Echeverría, s. f.).

En todos los anteriores casos de países del Magreb se han dado tres factores comunes preponderantes que explican avance que ha tenido el fenómeno terrorista, un primero sería el inicio del secuestro de 
turistas (Echeverría, s. f.) en estas zonas, lo cual ha ayudado a la financiación de las organizaciones terroristas y a la difusión del terror. Un segundo factor es el inicio de los ataques suicidas que se dan primeramente en Argelia y se dispersan por el resto de los países (Echeverría, s. f.), y que dan como resultado que se convierta en un método común en el terrorismo, además de que les permite un incremento del número de víctimas. Como tercer factor se encuentra, más recientemente, el papel jugado por los medios de comunicación (Echeverría, s. f.) para dar a conocer a la opinión pública la letalidad de algunos grupos terroristas, que en ocasiones son los que transmiten mensajes o videos de muertes de los grupos terroristas.

Todos los anteriores antecedentes del terrorismo y las acciones conseguidas por los otros grupos terroristas en la región africana permiten ver que estas nuevas células terroristas nacen con una mayor letalidad, con ideologías cada vez más extremas y con una capacidad de actuar y ganar adeptos, en muchos lugares del mundo, mayor a la que podían tener hace veinte o treinta años.

\section{Instituciones de seguridad dentro de la Unión Africana}

La institucionalización de la integración africana inicia con la primera organización creada, la Organización para la Unidad Africana (OUA) en 1963, la cual tenía como principales objetivos la promoción de la unidad y solidaridad de los Estados africanos, la solución pacífica de conflictos, así como la defensa de su soberanía y la erradicación del colonialismo.
Entre los años 1999 y 2002 se dio un proceso de transición de la OUA a la Unión Africana (UA), esta nueva institución continúa con el legado de su predecesora; pero con una serie de modificaciones que buscaban dar una solución a las falencias de la OUA. La UA se vio principalmente fortalecida por el establecimiento del derecho de intervención en los asuntos internos de los Estados miembros en casos de crímenes de guerra, genocidios y otros crímenes contra la humanidad, con base en la decisión tomada por la Asamblea de la UA con una mayoría de dos tercios. Otro de los principios es el establecimiento de una política de seguridad en conjunto para el continente africano, además de la inclusión de la promoción de la democracia (Moller, 2009).

La renovación del sistema integracionista responde a la necesidad de cambio en la OUA, debido a que esta presentaba un desgaste en su funcionamiento. Con la creación de la Unión Africana, se han mostrado reformulaciones acerca de la importancia de mantener la paz y la seguridad regional. La diferencia se puede observar en su carta fundacional, ya que “...establece el derecho de la UA a la intervención en otros Estados miembros en casos de 'graves circunstancias'" relacionadas con genocidios, crímenes contra la humanidad o crímenes de guerra" (González, 2013) temas que para la OUA no eran relevantes, e incluso predominaban los principios de no intervención y soberanía de los países.

Al cambiar las necesidades y aumentar la inseguridad en la región, el organismo, de acuerdo con sus falencias y a las fallidas 
intervenciones de las Naciones Unidas en conflictos en el pasado, evolucionó para poder prevenir y sobrellevar esas situaciones; es por esta razón que se establecen las instituciones encargadas de la seguridad: "El acta constitutiva establece diecisiete instituciones para abordar la seguridad y el desarrollo continental" (Tjiurimo, 2013).

Las instituciones delegadas a la seguridad del continente se sitúan en una estructura conocida como la Arquitectura de la Paz y Seguridad en África (APSA), la cual tiene, como prioridad, establecer una mejor coordinación entre los países y entre los bloques comerciales regionales, con el fin de prevenir conflictos así como de gestionar las situaciones que ya acontecen en el continente; esto se debe a que es más favorable planear la prevención de conflictos, ya que no es posible manejar por completo los problemas que enfrenta hoy en día África, debido a que son de carácter histórico así como religioso y étnico, temas que poseen un alto grado de complejidad para ser tratados.

Además, APSA consiste en un marco de consolidación de la paz que incluye un "Panel de sabios" para promover los esfuerzos de mediación; una fuerza de reserva africana de reacción rápida anclada en cinco brigadas regionales; un Comité de Estado Mayor; un Fondo de Paz; y un sistema continental de alerta temprana (Tjiurimo, 2013).

El financiamiento de APSA se mantiene por medio de los mayores países miembro, como Nigeria, Sudáfrica, Argelia, Libia y Egipto; no obstante, este depende del financiamiento del exterior; en específico, de la Unión Europea “....depende casi en un 90\% de la financiación exterior, especialmente de la Unión Europea” (González, 2013).

Ahora bien, una ambiciosa iniciativa por parte de la UA ha sido la creación del Consejo de Paz y Seguridad, en el 2004 y gracias a este se han desarrollado otras instituciones de igual importancia como “...el Sistema Continental de Alerta Temprana, el Panel de Sabios, la Fuerza Africana de Estabilización (FAE) y la Política Común Africana de Defensa" (González, 2013). Aunado a esto, también se pueden mencionar los esfuerzos en materia de instrumentos como la Declaración de Cambios Inconstitucionales de Gobierno, con el fin de rechazar y evitar que se den golpes de Estado “... entre 2003 y 2012 se produjeron 12 golpes de Estado, y la UA suspendió a ocho países de su participación en el organismo, como sanción por la forma irregular de cambio de gobierno" (González, 2013). A pesar de la falta de medidas más fuertes por parte de la UA hacia los Estados, se debe reconocer el esfuerzo en materia de estabilidad política; asimismo, la Carta Africana de Derechos Humanos y acerca de los derechos de las mujeres también son propuestas importantes en el marco de la integración africana.

Si bien los avances que ha tenido la UA con respecto a la seguridad han sido notorios, el hecho de que dependa financieramente de organizaciones externas al continente y no de sus propios miembros, propician que se involucren con gran relevancia actores tales como la Organización de Naciones Unidas y la Unión Europea, razón por la cual deben ser mencionados como parte importante del desarrollo de la seguridad de la Unión Africana. 
En relación con la estabilidad política, la Organización de Naciones Unidas se ha dado a la tarea de cooperar de manera conjunta con la Unión Africana para mantener la seguridad regional: "Es obvio que la ONU y la UA deben cooperar estrechamente para ofrecer seguridad en África" (Tjiurimo, 2013). Dentro de la labor conjunta de la ONU con la Unión Africana se encuentra el Programa de 10 años de fortalecimiento de capacidades para la UA, el cual tiene como objetivo la mejora de las capacidades tanto de la Unión Africana como de los sub- regionalismo africanos, para trabajar en conjunto con la ONU y poder actuar ante los retos de la seguridad humana (NEPAD, s. f.). El foco inicial del trabajo conjunto era la seguridad regional; no obstante, se involucran también temas de gobernanza y desarrollo.

Con respecto a la relación con la Unión Europea, aparte del peso que representa en financiamiento para el organismo africano, históricamente estos dos continentes han tenido grandes vinculaciones. En referencia a materia de seguridad y defensa, la Unión Europea ha realizado:

...la Política Exterior de Seguridad Común (PESC)/Política Exterior de Seguridad y Defensa (PESD), donde el Consejo Europeo ha colocado el núcleo competencial de gestión de crisis, tanto humanitarias y de evacuación, como las de mantenimiento de la paz, incluido el envío de tropas y el desarrollo, en su caso, de operaciones militares. (Dersso, 2010, p. 37)

Para llevar esto a cabo, la UE tiene un Centro de Operaciones (OpsCentre) así como de Fuerzas de Reacción Rápida, "pero la UE ha querido también compartir la aspiración africana de ser gestora eficaz de sus propias crisis y cooperar con ella elaborando el Plan para el Fortalecimiento de las Capacidades Africanas para la Prevención, Gestión y Resolución de Conflictos..." (Dersso, 2010, p. 37).

Por estas razones así como por los problemas globales que se presentan, la Unión Europea tanto como la Unión Africana fortalece sus relaciones, ya que son vecinos y, por ende, las carencias en materia de seguridad que amenazan al continente africano resultan situaciones problemáticas latentes para Europa.

\section{Iniciativas para combatir el terrorismo en Somalia y Nigeria}

En este apartado se analizan los casos de estudio seleccionados, con respecto a Somalia se señalan las falencias que presenta el Estado en la generación de respuestas ante las diversas amenazas generadas por el grupo terrorista Al-Shabaaby se expondrá el papel que ha tenido la Unión Africana y la ONU y su intervención en el país a través de la misión especial AMISOM. Se presentan sus aciertos y desaciertos, así como el estado actual de la misión y los retos ante las recientes amenazas.

A su vez, el Estado nigeriano, aunque se toma en consideración como una de las economías más fuertes de África, que incluso ayuda en el financiamiento de la Unión Africana, con su situación interna con el grupo terrorista BokoHaram, desencadena una serie de problemas de seguridad a nivel interno. 


\section{Somalia}

\subsection{Principales amenazas: el grupo terrorista Al-Shabaab}

En Somalia, la principal amenaza en temas de seguridad es el grupo terrorista AlShabaab, el cual surgió a mediados de la década de los noventa (de la Corte, 2015). Sobre su ideología, si bien al inicio mantuvo una ideología wahabí, a los pocos años de existir tuvo una gran transformación, influenciado por la invasión proveniente de Etiopía en el año 2006, la cual provocó que el grupo experimentara una "rápida radicalización en una doble vertiente nacionalista y religiosa" (de la Corte, 2015, p. 6).

La invasión de Etiopía tuvo un papel importante en el establecimiento de la organización en Somalia, ya que permitió a Al-Shabaab ganar simpatizantes al presentarse como un frente para expulsar a etíopes, además de defender el establecimiento de un Estado Somalí unificado y fortalecido (de la Corte, 2015). Es importante señalar que a partir del año 2007 fue cuando Al-Shabaab fortaleció su arista religiosa y desató una estrategia más agresiva y restrictiva hacia los territorios que controlaban, ya que desde ese año el grupo declaró como principal objetivo el establecimiento de un Estado que respetara las leyes del Islam además de fortalecer su presencia en la región con el fin de extender la yihad. Aunque es importante destacar que dentro del grupo se presentan divisiones significativas ${ }^{1}$.

1 Uno de los frentes declara que su objetivo principal es luchar contra el gobierno central de Somalia y contra las fuerzas extranjeras, como es el caso de AMISOM (misión de la Unión Africana). Otro grupo se encuentra influenciado por los grupos radicales del
Con respecto a las actividades económicas, como mencionan Masters y Sergie (2015), para obtener financiamiento el grupo ejecuta actividades como secuestros, donaciones de organizaciones extranjeras y extorsión a empresas locales. Además, han elaborado una red de contrabando de azúcar hacia Kenia, y presentan indicios de cooperación con la piratería desarrollada en las costas de Somalia.

Los principales territorios de Somalia que se encuentran bajo el dominio de Al-Shabaab se localizan en las regiones del sur y el centro del país (Mastersy Sergie, 2015). Sin embargo, es importante destacar que los esfuerzos para contener el avance del grupo, principalmente los que son liderados por la AMISOM, han tenido un impacto significativo ya que sí se ha logrado retomar regiones que se encontraban bajo el control total de militantes de Al-Shabaab, punto que se va a profundizar más adelante.

Finalmente, es necesario mencionar las acciones de este grupo fuera de las fronteras de Somalia, donde Kenia ha sido un foco principal. En este país, Al-Shabaab se ha aliado con grupos terroristas nacionales, como el Centro de la Juventud Musulmana (CJM), ubicado en Nairobi, quienes colaboraban con propaganda y envío de militantes hacia Somalia; incluso en el 2012 Al-Shabaab reconoció al CJM como parte de su organización. Esta alianza fue determinante para lograr ejecutar acciones con un impacto significativo dentro de Kenia, pues les permitió contar con apoyo dentro del propio país (de la Corte, 2015).

Golfo y las temáticas de carácter transnacional, como la expansión de la yihab hacia otros países (Masters y Sergie, 2015). 
Un ejemplo de este fue el ataque al centro comercial Westgate en Nairobi, capital de Kenia en el año 2013, acto que, de acuerdo con la BBC, tuvo como resultado la muerte de al menos 67 personas.

\subsection{Acción estatal de Somalia}

Para comprender el éxito que ha tenido este grupo terrorista en Somalia, es necesario brindar una breve perspectiva de la situación del Estado somalí para entender su vulnerabilidad y la incapacidad que presenta para lograr combatir y detener el avance de los diversos grupos terroristas que se encuentran en su territorio, en especial con Al-Shabaab.

Sobre la historia de Somalia, cabe destacar que un hecho decisorio ocurre en 1991, año en el que cae el régimen de Siad Barre quien fue dictador desde 1969 (Gutiérrez, 2011). Tras la caída de Barre se inició una etapa en la que diversos clanes alrededor del país declararon una guerra, lo cual provocó una guerra civil en el país y que generó lo que se podría denominar como un estado de anarquía; en el que el Estado perdió por completo el monopolio del uso de la fuerza.

Ramis (2013) describe que en el país "el poder político en Somalia es local, fragmentado, violento en algunos casos, heterogéneo y basado en estructuras híbridas de instituciones formales e informales controladas por clanes o milicias, en que interactúan diferentes órdenes sociales" (p. 2).

Debido a las condiciones de inestabilidad en las que se encontraba Somalia durante la primera década del 2000,el país no tuvo la capacidad de responder a la formación de estas organizaciones criminales y terroristas, ni tampoco fue capaz de responder a las invasiones de otros Estados (como la suscitada en el año 2006 por parte de Etiopía). Por tanto, estudios como los de la Corte Ibáñez señalan que, "Al-Shabaab no hubiera progresado como lo hizo hasta 2011 de no ser por la tardanza en articular una estrategia y una fuerza adecuadas para contrarrestar su empuje inicial" (de la Corte, 2015, p. 31).

\subsection{Intervenciones internacionales}

\subsubsection{Acciones multilaterales. El caso de la AMISOM}

La misión de la Unión Africana en Somalia (AMISOM) fue creada en el año 2007 en el seno de Consejo de Paz y Seguridad de la UA. Es una misión de paz de carácter regional implementada mediante la gestión de esta institución y con la aprobación de las Naciones Unidas. Es importante destacar que fue creada por un período de vigencia de seis meses; sin embargo, se ha ido renovando el mandato de forma constante con decisiones efectuadas en el Consejo de Seguridad de la UA, actualmente se encuentra vigente hasta el 31 de mayo del 2019. No obstante, el Consejo de Seguridad ha mencionado en distantes resoluciones -la última resolución 2431 en el 2018-el deber de disminuir las tropas armadas para el 28 de febrero del 2019.

Esta tiene como principal objetivo implementar condiciones para el establecimiento de un Estado somalí estable además de contener el avance del grupo Al-Shabaab. Se encuentra dividida dentro de cinco grandes áreas estratégicas: la policía de AMISOM, el trabajo humanitario, el componente militar, 
el componente civil y la marina de AMISOM. De forma general, cada una de estas áreas busca de diferentes maneras fortalecer a las estructuras y competencias del Estado de Somalia, con el fin de ir construyendo la estabilidad en el país a través de la transformación de las instituciones, para que estas sean capaces de prestar los servicios a la población somalíe y de así ganar la legitimidad ante su pueblo, así como "crear las condiciones necesarias para reconstruir ya alcanzar el desarrollo sostenible en Somalia" (AMISOM MissionProfile).

Un ejemplo de la visión que se intenta implementar es la policía de la AMISOM, la cual tiene como objetivo "entrenar, monitorear y dar consejo a la Fuerza de Policías de Somalia (SPF) con el propósito de transformarla en una organización creíble y efectiva" (AMISOM MissionProfile). Es decir, el objetivo de esta policía no se reduce a una respuesta armamentística al problema, sino que se trata de abordar como un acompañamiento para las fuerzas gubernamentales, con el fin de que a largo plazo sean capaces de implementar las acciones necesarias por sí mismas.

Actualmente, la misión cuenta con la colaboración Burundi, Camerún, Yibuti, Etiopía, Gambia, Ghana, Kenia, Níger, Nigeria, Senegal, Sierra Leona, Uganda, Zambia y Zimbawe. Sin embargo, la mayoría de los 22000 soldados de la AMISOM provienen de Uganda, Kenia, Burundi, Sierra Leona, Etiopía y Djibuti.

Sobre los alcances de la misión se puede señalar que el trabajo de las fuerzas militares provenientes de los países de la región, en conjunto las fuerzas armadas de Somalia, lo siguiente:
Ha permitido la ocupación en los dos últimos años todos los puertos y ciudades importantes del sur del país -el último Baraawe a principios de octubre de 2014-, así como el control gubernamental de la capital Mogadiscio, lo que ha privado al grupo Al-Shabab de su principal fuente de financiación basada en la extorsión económica. (Fuente, 2014, p. 10)

No obstante, el éxito de la misión no ha sido tan positivo en las zonas interiores de Somalia, ya que en estos lugares la presencia de Al-Shabaab continúa siendo significativa y con un vasto control de los territorios, por lo que se puede decir que "este grupo islamista mantiene su capacidad para golpear en el interior del territorio y en los países vecinos, principalmente en Kenia donde se ha convertido en el defensor del irredentismo de la minoría musulmana allí asentada" (Fuente, 2014, p. 12).

Es importante destacar el cambio de rol en el que se encuentra la AMISOM, pues, como ya se mencionó, en Somalia en el 2012 se estableció la elección de un nuevo gobierno federal, el cual ha declarado su interés por fortalecer las instituciones nacionales con el fin de no depender tanto de las acciones de terceros, llegando incluso a declarar que "la provisión de seguridad es la prioridad de su gobierno" (Williams, 2013).

Ante esta situación, la AMISOM enfrenta nuevas condiciones, en las que la prioridad debe ser darle un acompañamiento al nuevo gobierno, fortalecerlo y disminuir la dependencia del Estado somalí de las intervenciones de otros para mantener la relativa estabilidad en su territorio. 


\section{La Unión Europea}

La Unión Europea (UE) ha tenido una importante participación en Somalia, la cual inició en el 2010, cuando se aprueba una misión específica para este país. Esta misión busca coordinar esfuerzos con los diferentes actores que se encuentran en la región, como lo son AMISOM, la Unión Africana, Naciones Unidas y Estados Unidos, y esta se encuentra "dirigida a favorecer y orientar la reforma del sector de seguridad del país que la acoge como medio para incrementar su estabilidad" (de la Corte, 2015, p. 35).

El apoyo que ha dado de forma directa la UE a la AMISOM se refleja en la asistencia financiera con la cual se da el pago de las tropas de la misión; esto por medio del Fondo de Apoyo para la Paz en África (APF) del Fondo Europeo para el Desarrollo, y "ha contribuido con más de 600 millones de euros desde 2007 a la lucha de AMISOM contra Al-Shabaab" (Ferro, 2016, p.7).

Sin embargo, la participación de la UE en la misión ha sufrido importantes cambios, ya que en el año 2016 se estableció la disminución de los recursos para esta causa, lo cual implicó una reducción del $20 \%$ del total de los fondos. Esto ha tenido efectos significativos, pues, como mencionan Mahmood y Ani (2017), el pago de las tropas es enteramente dependiente de los recursos otorgados por la Unión Europea, por lo cual la disminución de estos fondos puede causar efectos significativos en el desarrollo de las acciones.

El recorte de los recursos realizado por la Unión Europea se puede deber a dos grandes causas: en primer lugar, debido al "agotamiento del donante", el que se puede entender por el creciente aumento de los recursos asignados por un largo período -más de 10 años- $\mathrm{y}$ a la responsabilidad de ser el único donante responsable por el pago de las tropas de la misión. En segundo lugar, esta decisión fue tomada por el cambio en las prioridades de la UE para el continente, donde la temática de los crecientes flujos migratorios domina la agenda, así como el objetivo de confrontar amenazas como BokoHaram, además de los distintos intereses geopolíticos de cada país europeo en África (Mahmood y Ani, 2017).

\subsubsection{Acciones bilaterales}

\section{Kenia}

Kenia juega un rol fundamental, ya que presenta múltiples factores que involucran al país dentro de la dinámica de Al-Shaba$\mathrm{ab}$, lo cual repercute en sus relaciones con Somalia. Un primer factor para identificar esta alta correlación entre ambos Estados es el hecho de ser vecinos, razón por la cual se presenta una mayor vulnerabilidad ante las acciones que surjan a partir del alto grado de inestabilidad de Somalia; además se ser receptor de una gran cantidad de migrantes.

De igual forma, como ya se ha mencionado, Al-Shabaab se ha organizado y realizado alianzas con grupos musulmanes como el Centro de la Juventud Musulmana, lo cual permitió la coordinación en una serie de atentados, sin olvidar que Kenia ha sido uno de los focos principales de las iniciativas de Al-Shabaab, con el fin de internacionalizarse y de luchar por la formación de gobiernos de carácter islámico. 
Ante esta situación, en el año 2011, el gobierno keniano decidió realizar una intervención militar al sur de Somalia, es decir, en los territorios transfronterizos con Kenia; para llevar a cabo un ataque hacia Al-Shabaab para detener su avance y controlar el estado de su frontera norte (Fuente, 2014), aunque el motivo oficial fue una respuesta hacia el secuestro de visitantes de España, lo cual afectaba el turismo del país.

Como señala Fuentes, los resultados de esta invasión no fueron los esperados, ya que Al-Shabaab realizó una guerra de guerrillas, para lo cual Kenia no estaba preparada, de forma que la intervención, que se esperaba corta, se prolongó. Ante la incapacidad de responder a las variantes, Kenia tuvo como única salida incorporarse a los esfuerzos de la AMISOM.

\section{Etiopía}

Como ya se había mencionado anteriormente, en el año 2006 las fuerzas militares de Etiopía realizaron una intervención en el país, lo cual causó una fuerte oleada de nacionalismo en Somalia ante la invasión de agentes extranjeros. De este sentimiento se aprovechó Al-Shabaab para ganar militantes en sus frentes y legitimizarse ante el pueblo de Somalia.

Como señala Williams (s. f.), el objetivo de esta invasión fue tratar de instalar un nuevo régimen de carácter más democrático y estable, con el fin de solucionar el estado de crisis presente en Somalia. Sin embargo, este esfuerzo tuvo grandes complicaciones, debido a que, como ya se mencionó, la población somalíe no lo vio de forma positiva, sino que lo caracterizaba como un producto de los intereses de actores externos al país.

Además, esta intervención fue tomada con escepticismo en la Unión Africana con respecto a la legitimidad y efectividad de las acciones de Etiopía, principalmente por la falta de una estrategia de paz para el país, que produjeron un ambiente de violencia y caos (Williams, s. f.).

La salida de la intervención etíope ocurrió en el 2009, pero hay que tomar en cuenta que en el 2007 se creó la AMISOM, ante lo cual posteriormente se incluyó el país en esta estrategia de carácter regional.

\section{Nigeria}

\subsection{Principales amenazas: BokoHaram}

El problema de seguridad en Nigeria no es nuevo, una de las zonas más afectadas por ello es la región de Delta del Níger por grupos criminales que se dedicaban a atacar las instalaciones petroleras y a los secuestros, después de que la situación en la región mejoró debido a "la puesta en marcha del Programa de Amnistía de la administración Yar'adua-Jonathan y el programa de desmovilización, la inestabilidad del país está marcada en la actualidad por la emergencia del terrorismo fundamentalista en el Norte del país" (Ministerio de Relaciones Exteriores y Cooperación, s. f.).

El grupo terrorista del que se habla desde hace algunos años es el BokoHaram en Nigeria, que inicia en Maiduguri "de la mano del mullah Mohamed Yusuf, quien quería imponer la Sharía para 'limpiar al país de la educación occidental'. En el momento 
del lanzamiento del embrión del grupo, en 2002, Yusuf lo denomina Compañeros del Profeta" (Echeverría, 2014, p. 4). Posteriormente, con la adopción del nombre BokoHaram cambia a "la educación occidental es pecado" en hausa (Ministerio de Relaciones Exteriores $Y$ cooperación, s. f.). Este grupo terrorista tuvo un radio de acción en el noreste del país en sus inicios, aunque el Lago de Chad (las provincias de Adamawa, Borno y Yobe) continúa como la zona más afectada, (Mahmood, 2018). El año 2015 se marca como su periodo de mayor auge, ya que, por su capacidad de acción fortificada, sus métodos cambiaron para ser más violentos y más eficaces en cuanto al deceso de sus objetivos, además "los ataques armados contra las comunidades rurales pasaron de ser ataques de "golpe y huida" a ser ocupaciones militares del territorio en las que se administra la seguridad, la justicia y los recursos económicos" (Ministerio de Relaciones Exteriores y Cooperación, s. f.).

El problema con BokoHaram no solo afectó al noreste del país sino su radio de acción fue expandido incluso a regiones como Chad, Níger y Camerún, aunque no les fue posible llevar a cabo ataques en zonas más allá de estas (Mahmood, 2017). Según los datos de Instituto de Estudios de Seguridad, la región del Lago de Chad es la más golpeada por el BokoHaram: se reportaron 280 ataques en 2016, 362 en 2017 cifras menores a los 392 ataques reportados en 2015 (Mahmood, 2018), el $60 \%$ del total de los ataques ocurrió en las provincias del norte de Nigeria (Adamawa, Borno y Yobe) (Mahmood, 2018), esto sucede principalmente porque se ha dado un "aumento de la violencia en los estados de Borno y Adamawa, en lugar de una disminución de los incidentes en Camerún, que se mantuvo estable. Los ataques en Borno aumentaron de 120 en 2016 a 189 en 2017, mientras que Adamawa enfrentó un salto similar de siete en 2016 a 26 en 2017" (Mahmood, 2018).

Actualmente, el BokoHaram enfrenta serias transformaciones internas, que se han gestado desde un par de años atrás, cuando en agosto de 2016 el grupo se separó en dos fracciones: Jama'atuAhlis Sunna Lidda'awatiwal-Jihad (JAS) y IslamicState West Africa (ISWA) (Mahmood, 2018). La separación se dio a raíz de discrepancias sobre sus potenciales víctimas; una fracción proponía el asesinato de la población musulmana, lo que originó el conflicto. "El líder de mucho tiempo AbubakarShekau favorece un perfil de ataque más indiscriminado, mientras que la nueva facción de Abu Musab al-Barnawi respaldada por el Estado Islámico prefiere involucrar directamente a las fuerzas de seguridad" (Mahmood, 2017).

Existe una evolución en cuanto al empleamiento de acciones terroristas de BokoHaram en el tiempo, cuando en 2014-2015 se ejecutaban ataques suicidas en 2016-2017 su método cambió a utilizar más a las mujeres en sus ataques suicidas hasta enviar a más de un combatiente suicida, los ataques suicidas fueron la mitad de todos sus golpes y el 41\% fueron ataques suicidas en 2017 (Mohmood, 2018); sin embargo, se debe apuntar que se registran progresos en años recientes: "casi la mitad de todos los ataques suicidas de BokoHaram en 2016 pueden describirse como infructuosos, ya que solo murió el atacante suicida o se le impidió alcanzar su objetivo" (Mahmood, 2017). 
Otro cambio importante se ha dado en cuanto a la letalidad de los ataques por BokoHaram. Mientras que entre los años 2014-2015 todos sus golpes terminaban en una importante cantidad de muertes, a partir de 2016 la tendencia varía. A pesar de que aún ocurren una cantidad considerable de ataques, estos no resultan con cientos de bajas (Mahmood, 2017), lo cual se comprueba con los datos: "se registraron cerca de 1500 muertes en 2017, cerca de la de 2016 (a pesar del aumento en el número de ataques), y significativamente más bajas que las más de 5000 registradas en 2015" (Mahmood, 2018).

A pesar de que Nigeria es uno de los países que presta colaboración a favor de la lucha antiterrorista en Somalia, su condición interna no es del todo buena: un $61 \%$ de la población en el norte del país vive en condición de pobreza, por lo que se puede apuntar que "la volatilidad del estado Nigeriano es más compleja y abarca una serie de elementos, como, las tensiones étnicas y religiosas, y los altos niveles de desigualdad económica" (ConflictPrevention and RiskAnalysisDivision, s. f., p. \#). Son estas mismas condiciones de pobreza y desigualdad social lo que lleva, como se mencionó en apartados anteriores sobre el contexto social, a la proliferación de células terroristas que se generan principalmente por el descontento ante la exclusión del gobierno.

Una característica sobresaliente sobre el terrorismo en Nigeria y específicamente el aplicado por el BokoHaram contiene un fuerte componente de odio religioso hacia los grupos católicos en la región norte de Nigeria, debido a los repetidos ataques hacia templos católicos, a pesar de que las autoridades nigerianas lo nieguen,
These persistent attacks are likely to deteriorate relationship between Christians and Muslims within the North and with the South. Most Northern political elites (recently reinforced by the Governor of Kaduna) continue to deny the religious dimension to the attacks by Boko Haram (Conflict Prevention and Risk Analysis Division, s. f.).

\subsection{Acción estatal de Nigeria}

En el caso del accionar estatal de Nigeria en contra de BokoHaram, este se ha concentrado en la región norte, es decir, en las provincias fronterizas del Lago Chad, donde existen precedentes de la escasa presencia institucional del gobierno nigeriano. El mayor peso sobre el combate a la amenaza del BokoHaram ha recaído en el gobierno de Nigeria, debido a las "raíces y su actividad predominante en el país. Sin embargo, la expansión continua del grupo demuestra de manera pertinente la interconexión a través de los límites de la región del Lago Chad" (Mahmood y Ani, 2018, p.10).

Un punto de inflexión en el combate al grupo terrorista se dio con la elección del presidente MuhammaduBuhari en 2015, quien intenta renovar las relaciones con los países vecinos al Lago Chad, con el fin de trabajar de manera conjunta en una respuesta, "visitando Níger y Chad en sus primeros viajes al exterior para galvanizar el apoyo a una respuesta de colaboración a la amenaza BokoHaram" (Mahmood y Ani, 2018, p.10). A pesar de las operaciones constantes para desarticular al BokoHaram de las regiones en que opera, los resultados no son aún satisfactorios: "BokoHaram en su conjunto ha sido sistemáticamente subestimado públicamente 
por la seguridad nigeriana y los funcionarios del gobierno desde su surgimiento, una tendencia que continúa con la división del movimiento en dos grupos" (Mahmood y Ani, 2018, p. 14).

Desde agosto de 2016 hasta mayo de 2018 se han llevado a cabo alrededor de diez operaciones militares por parte del gobierno nigeriano, centradas en dos asuntos: la recuperación de los territorios de población urbana en las provincias de norte del país y la seguridad de las rutas de transporte (Mahmood y Ani, 2018), aunque la estrategia de recuperación de territorios no ha alcanzado a las zonas rurales, por ejemplo, en la provincia de Borno continúan en su estado de inseguridad (Mahmood y Ani, 2018). Respecto a la seguridad de las rutas de transporte, se logró con la "reapertura de la carretera Maiduguri-Banki en marzo de 2018. Sin embargo, similar al enfoque en los centros urbanos, los militantes siguen siendo capaces de realizar ataques a lo largo de las rutas reabiertas" (Mahmood y Ani, 2018, p. 12).

Las operaciones militares por parte del gobierno de Nigeria se han conducido desde dos vías: las operaciones terrestres y las operaciones aéreas; conjuntamente estas operaciones han tenido éxito en diferentes zonas del país: "las fuerzas terrestres han sido un aspecto importante de la respuesta militar, particularmente en el centro-sur de Borno, donde la Operación Deep Punch I y II ha visto una serie de incursiones en el área del bosque de Sambisa" (Mahmood y Ani, 2018, p. 12). En relación con las operaciones aéreas y su importancia para el combate hacia el grupo terrorista en la zona de Borno, "incidentes trágicos como el bombardeo accidental de un campamento de desplazados internos en Rann en enero de 2017 demuestran los riesgos detrás de este enfoque" (Mahmood y Ani, 2018, p. 12).

Otro aspecto de resaltar en cuanto al combate a las fracciones del grupo BokoHaram es la relevancia mediática que ha caracterizado a la fracción JAS, ya que sus ataques son más frecuentes aunque menos letales en cuanto a la pérdida de vidas humanas, por tanto, la milicia nigeriana se ha concentrado en su combate (Mahmood y Ani, 2018). Sin embargo, recientemente se han llevado a cabo operaciones en territorios que ocupa la fracción de ISIS-WA: "a fines de abril de 2018, el ejército nigeriano anunció OperationLastHold, que se extendería a partir de mayo hasta agosto. El objetivo es limpiar el lado nigeriano del Lago Chad" (Mahmood y Ani, 2018, p. 14).

\subsection{Intervenciones internacionales}

En cuanto a la cooperación del exterior hacia la lucha contra el terrorismo no se ha dado una verdadera estrategia conjunta, y es muy recientemente que se ha venido pensando que la amenaza del grupo terrorista BokoHaram es global y no solo de Nigeria. Específicamente la Unión Europea ha expresado que Francia podría cooperar; sin embargo, hasta el momento no se ha llevado a cabo ninguna acción. Se dice que "France couldprovidethelogisticalsupport (drones) thatNigeriansecurityservicesneed in theirfightagainstthe radical Islamisminsurgents [traducción]" (Zounmenou, 2014). Una acción concreta que vino del exterior, directamente del Banco Mundial, fue la donación de 2.1 
billones de dólares para la lucha contra el terrorismo con un interés muy bajo y con un tipo de 40 años para ser cancelado. Dentro de Nigeria ha causado gran revuelo, por considerar que el Banco Mundial puede y va a opinar respecto a cómo pueden gastar el dinero. De manera muy certera el Banco Mundial apunta:

If the money is spent wisely - on health, education, infrastructure and restoration projects in the north-east - this might begin to address some of the underlying factors which have allowed Boko Haram to flourish, making it one of the most potent weapons in the Nigerian government's arsenal (Simon, 2015).

\subsubsection{Acciones multilaterales}

Las acciones regionales para combatir al grupo terrorista BokoHaram se han centrado primordialmente en el uso del recurso militar (Mahmood y Ani, 2018); ahora, la cooperación regional militar para combatir a BokoHaram tiene sus inicios en 2012, cuando las naciones de Nigeria, Chad, Níger, Camerún y posteriormente Benín se unieron para dar una respuesta conjunta a la amenaza terrorista; en este año la Multinational Security Force recibió el mandato de combatir al grupo terrorista, en 2014 esta unidad pasó a llamarse Multi- NationalJointTaskForce (MNJTF) (Sawadogo, 2017). Desde el inicio del gobierno del presidente Buhari, la cooperación militar contra el terrorismo se vio revigorizada, como se mencionó anteriormente (Mahmood y Ani, 2018). Por parte del Consejo de Paz y Seguridad de la Unión Africana, en 2016 se renovó el mandato de MNJTF por doce meses más (Sawadogo, 2017). Un punto importante por resaltar es que "la línea divisoria entre la acción militar nacional y el MNJTF también puede difuminarse, y los ejércitos nacionales de cada país realizan operaciones anti-BokoHaram fuera de los confines del MNJTF" (Mahmood y Ani, 2018, p. 11).

En 2016 se realizaron dos importantes acciones contra el grupo terrorista a cargo de las operaciones de Gama Aiki y RawanKada. La operación Gama Aiki se concentró en los territorios de Baga en Nigeria, Baga-Sola en Chad, Diffa en Níger y Mora en Camerún (Sawadogo, 2017); la localización geográfica de "las operaciones en el área del Lago Chad y la frontera Níger-Nigeria sugiere que el MNJTF ha estado lidiando con ISIS-WA, en lugar de las operaciones conjuntas Camerún-Nigeria que se han dirigido a JAS más al sur" (Mahmood y Ani, 2018, p. 11).

Operaciones recientes de la MNJTF se han enfocado en la unión de las militares de Nigeria y Camerún, los cuales ejecutan operaciones de maneja conjunta en ambos territorios (Mahmood y Ani, 2018), no obstante, a pesar de la cooperación regional militar entre los países vecinos a Nigeria, las relaciones entre estos continúan tensas, "evidencia de esto incluye la persistencia de acciones descoordinadas por parte de los estados, y declaraciones de triunfo contra BokoHaram por parte de países individuales durante operaciones conjuntas" (Sawadogo, 2017). La MNJTF como la mejor alternativa regional para desarticular a BokoHaram enfrenta serias amenazas, tales como las financieras, surgidas desde la conformación de esta unidad militar que se ve agravada actualmente por los problemas económicos 
que afrontan sus dos más grandes socios militares, Nigeria y Chad (Sawadogo, 2017): "las contribuciones de Nigeria, el Reino Unido, la Comunidad de Estados Sahelosaharianos (CEN-SAD) y la Unión Europea no son suficientes para cubrir el presupuesto inicial, estimado en $700 \mathrm{mi}$ llones de dólares" (Sawadogo, 2017). Finalmente, desde el punto de vista del éxito del MNJTF, existen cuestiones que deben mejorar, por ejemplo, en lo que se refiere a su eficacia, el Foro de Dakar sobre Paz y Seguridad en África recomendó cuando se reunió los días 5 y 6 de diciembre de 2016 , con un llamado para que las fuerzas de defensa y seguridad se adapten a las amenazas actuales.

\section{Consideraciones finales}

Uno de los problemas que se da en la determinación de una línea de tiempo del terrorismo es que los primeros actos terroristas no eran considerados como tales, debido a que se analizaban desde otras perspectivas. En un primer momento, estos grupos terroristas se tomaron posiblemente como grupos insurgentes y sus actividades de bajo alcance no tenían la difusión que los medios de comunicación les dan hoy. Con los cambios introducidos con la globalización, en el sistema actual cualquier acto, por pequeño que sea, recibe gran cobertura, por lo que, a nivel internacional, los gobiernos y la población civil están muy al corriente de lo que sucede en cuanto al terrorismo se refiere.

La expansión del fenómeno del terrorismo se da por el cambio en la concepción que tienen estos grupos de quién es el enemigo. La imposición de gobiernos democráticos trajo como consecuencia la proliferación de más células terroristas, que buscan expulsar a los grupos aliados de occidente de los gobiernos africanos y así imponer su ideología religiosa dentro del sistema de gobierno.

El crimen organizado ha jugado un papel trascendental en el progreso de los grupos terroristas, debido a que la entrada de recursos constantes permitió la diversificación de las actividades de los grupos insurgentes; contaron, por ejemplo, con el capital suficiente para agrandar su área de acción, dentro de lo que se cuenta la compra de armas, dedicación exclusiva de los hombres a actividades terroristas, compra de propiedades para entrenamiento, establecimiento de terroristas en otros países de la región. A pesar de la discusión de los grupos terroristas extremistas sobre la moralidad del dinero proveniente del crimen organizado, este fue el factor determinante que les permitió desarrollarse.

Por otra parte, la Unión Africana, en su carta constitutiva, marca pautas para la prevención de golpes de Estado que anteriormente no se habían tomado en consideración por parte de la OUA, así como la creación de un consejo de paz y seguridad. Esto ha demostrado la necesidad del continente de tomar medidas para frenar el violento desarrollo del terrorismo en sus países, así como la necesidad de un organismo más sólido y renovado para los problemas que se agravan y que no permiten que la región se desenvuelva bien tanto a lo interno de África como con otras regiones.

Las instituciones que tienen incidencia en la UA en materia de seguridad constituyen 
buenas iniciativas, pero la falta de recursos económicos, así como de solidez política en los Estados miembro repercuten en que no se logre avanzar como se desearía en materia de seguridad. Aunado a esto, el hecho de luchar contra algo intangible como el terrorismo se vuelve un reto que, incluso para organismos de integración como la Unión Europea, es poco controlable. Por tanto, se analizó que la Unión Europea trata de colaborar con el continente vecino para evitar la propagación de actos terroristas en sus territorios.

Ahora bien, la Unión Africana es un organismo relativamente nuevo, por lo que la ayuda por parte de otros actores resultó de mucha utilidad para poder iniciar con la lucha contra el terrorismo. No obstante la visión de una UA, que se sostenga por sí misma suele ser complicado a corto plazo, ya que cuando se considera que se ha mejorado en materia de seguridad, un acto terrorista se manifiesta y es visto como una falla en la región, lo que deja en tela de duda la efectividad del organismo.

Sobre el papel que ha desarrollado la UA en la lucha por la paz y seguridad del continente, es fundamental el aumento de capacidades que ha tenido en comparación con la OUA. Sin embargo, presenta cierta vulnerabilidad ante los agentes externos, debido a las dificultades en aspectos como la obtención de recursos y financiamiento. A pesar de esto, el papel que ha logrado implementar en las iniciativas lideradas, como el caso de la AMISOM en Somalia, logra ejemplificar que sí ha mantenido su liderazgo de forma efectiva, con un aporte extra a los procesos de intervención que suceden en África.
Por ejemplo, en el caso de Somalia, es fundamental el cambio de enfoque que desarrolló, ya que la misión no se limitó a la lucha contra el terrorismo, sino que paralelamente se continúa implementando el fortalecimiento de las capacidades del Estado somalí, con el fin de que este logre conseguir una mayor estabilidad y logre por sí mismo combatir las problemáticas a las que debe enfrentarse. La iniciativa que ha liderado la UA realmente se ha enfocada en la construcción de un Estado de Somalia en el que se dejen atrás los fantasmas que lo rodean sobre la denominación de Estado fallido.

\section{Referencias}

BBC. Nairobi Westgate attack: Thevictims. Disponible en http://www.bbc. com/news/world-africa-24195845

Conflict Prevention and Risk Analysis Division. (s. f.). Nigeria: Continuing Boko Haram Attacks and the Heightened state of Insecurity. Disponible en https://www. issafrica.org/futures/publicationarchives/cpra-daily-briefing/ somalia-sudan-and-nigeria

De la Corte, L. (2015). Al Shabaab en el Cuerno de África. Instituto Español de Estudios Estratégicos. Disponible en http://www.ieee.es/Galerias/fichero/docs_investig/2015/ DIEEEINV05-2015_Al_Shabaab_ CuernoAfrica_LuisdelaCorte.pdf

Dersso, S. (2010). The role and place of the African Standby Force within the African Peace and Security Architecture. INSTITUT D'ETUDES DE 
SÉCURITÉ. Disponible en https:// www.issafrica.org/uploads/209.pdf

Echeverría, C. (s. f.). La colonización terrorista en África: El yihadismo africano (pp. 247-312). Disponible en http://www.Dialnet.com/Dialnet-LaColonizacionTerroristaEnAfrica-4545120.pdf

Ferro, J. (2016). Seguridad y desarrollo: La intervención de la Unión Europea en África. Documento de Opinión 29/2012. Instituto Español de Estudios Estratégicos. Disponible en http://www.ieee.es/Galerias/fichero/docs_opinion/2016/ DIEEEO29-2016_UnionEuropea_ SOMALIA_JoaquinFerro.pdf

Fuente, I. (2014). Somalia: Radiografía de un Estado frágil. Instituto Español de Estudios Estratégicos. Disponible en http://www.iee.es/Galerias/fichero/docs_analisis/2015/ DIEEEA06-2015_Somalia_RadiografiaEstadoFragil_xIIx_IFC.pdf

González, M. (2013). La Unión Africana: $¿$ ¿n nuevo actor global en paz y seguridad? Instituto de Estudios sobre Conflictos y Acción Humanitaria. Disponible en http://www.iecah. $\mathrm{org} / \mathrm{web} /$ index.php?option=com content $\&$ view $=$ article $\&$ id $=2278$ :launion-africana-iun-nuevo-actorglobal-en-paz-y-seguridad\&catid= 15: articulos\&Itemid $=9$

Gutiérrez, I. (2011). La Somalia de hoy y la defunción del Estado: La consolidación de un fracaso histórico. Relaciones Internacionales, 28, 11-31. Disponible en http://www. relacionesinternacionales.info/ojs/ article/view/309.html

Institutos Español de Estudios Estratégicos (IEEE) e Instituto Militar de Documentación, Evaluación y Prospectiva de Argelia (IMDEP). (2013). Tráficos de drogas en África Subsahariana. Disponible en http://www. ieee.es/Galerias/fichero/docs_trabajo/2013/DIEEET01-2013_IEEE IMDEP.pdf

Mahmood, O. (2017). Boko Haram in 2016: a highly adaptable foe. Institute for Security Studies. Disponible en https://issafrica.org/ iss-today/boko-haram-in-2016-ahighly-adaptable-foe

Mahmood, O. (2018). Despite its divisions Boko Haram is nl weaker. Institute for Security Studies. Disponible en https://issafrica.org/iss-today/despite-its-divisions-boko-haram-isno-weaker

Mahmood, O., Ani, N. (2017). Impact of EU funding dynamics on AMISOM. Institute for Security Studies. Disponible en https://issafrica. s3.amazonaws.com/site/uploads/ ear16.pdf

Mahmood, O., Ani, N. (2018). Responses to Boko Haram in the Lake Chad Region: Policies, Cooperation and Livelihoods. Institute for Security Studies. Disponible en https:// issafrica.s3 .amazonaws.com/site/ uploads/2018-07-06-research-report-1.pdf

Masters, J., Sergie, M. (2015). Al-Shabaab. Council on Foreign Relations. 
Disponible en http://www.cfr.org/ somalia/al-shabab/p18650

Ministerio de Relaciones Exteriores y Cooperación. (s. f.). Nigeria. Gobierno de España. Disponible en http://www.exteriores.gob.es/ Documents/FichasPais/Nigeria_ FICHA\%20PAIS.pdf

Moller, B. (2009). The African Union as a security actor: African solutions to African Problems? Research Centre working papers series 2, 57. London School of Economics and Political Science, London, United Kingdom. Available in https://www.eprints. lse.ac.uk/28485/

NEPAD. (s.f.). The Ten-Year Capacity Building Programme for the AU: Where we are and the way forward. Disponible en http://www. nepad.org/system/files/The $\% 20$ Ten-Year\%20Capacity\%20Building\%20Programme $\% 20$ for $\% 20$ the \%20AU.pdf

Ramis, N. (2013). El papel de la Unión Africana en Somalia: ¿Cuál es el futuro de la operación de paz de la AMISOM? InstitutCatalà Internacional per la Pau. Disponible en http://icip.gencat.cat/web/.content/ continguts/publicacions/policypapers/2013/arxius/policy_paper_08_ castellano.pdf

Sawadogo, W. (2017). Can the joint task force against Boko Haram stay the course?. Institute for Security Studies. Disponible en https://issafrica.org/iss-today/can-the-jointtask-force-against-boko-haramstay-the-course
Simon, A. (2015). THINK AGAIN: Could a long-term solution to Boko Haram come from the World Bank? Institute for Secury Studies. Disponible en https://www.issafrica.org/iss-today/ think-again-could-a-long-term-solution-to-boko-haram-come-fromthe-world-bank

Sitio web de la AMISOM, Unión Africana. (s. f.) Disponible en http://amisom-au.org/amisom-background/

Tjuimoro, A. (2013). The African Union and Security. Council of Councils. Disponible en http://www.cfr.org/ councilofcouncils/global_memos/ p32415

Pérez, A. (2004). África en la lucha antiterrorista. Real Instituto Elcano. Disponible en http://www.realinstitutoelcano.org/wps/portal/rielcano/ contenido?WCM_GLOBAL_CNTEXT=/elcano/elcano_es/zonas_es/terrorismo+internacional/ ari+174-2004

Williams, P. (2013). AMISOM in transition: The future of the African Union Mission in Somalia. Disponible en http://riftvalley.net/publication/ amisom-transition\#.VjIKddIveWh

Williams, P. (s. f). AMISOM's Five Challenges. Disponible en http://csis. org/story/amisom\%E2\%80\%99sfive-challenges.

Zounmenou, D. (2014). Nigeria's fight against Boko Haram: how can France help? Institute dor Security Studies. Disponible en https://www. issafrica.org/iss-today/nigeriasfight-against-boko-haram-how-canfrance-help 
